\title{
Fully Distributed Consensus Control for Linear Multi-Agent Systems: A Reduced-Order Adaptive Feedback Approach
}

\author{
Xianwei Li, Member, IEEE, Fangzhou Liu, Student Member, IEEE, Martin Buss, Fellow, IEEE, and \\ Sandra Hirche, Senior Member, IEEE
}

\begin{abstract}
This paper is concerned with fully distributed consensus control of linear multi-agent systems with undirected graphs. Two kinds of reduced-order adaptive output-feedback protocols are proposed. For the edge-based protocol, each edge is adapted by a scalar that is determined by the output information of the associated two agents; for the node-based protocol, each agent multiplies the connecting weights by a scalar that is determined by the relative output information of all neighbouring agents. Sufficient conditions in terms of the solvability of some matrix equations are derived for the existence of the two protocols. Furthermore, a tractable algorithm is constructed for designing the protocol gains. Compared with the existing related results, the proposed protocols have the following three merits simultaneously: of lower dimension, using relative output information about neighbouring agents and in the fully distributed fashion. A simulation example on formation flying of spacecrafts is presented to illustrate the efficacy of the proposed method.
\end{abstract}

Index Terms-Consensus, multi-agent systems, output feedback, reduced order, adaptive control.

\section{INTRODUCTION}

$\mathbf{M}$ ULTI-AGENT systems (MASs) have drawn considerable attention in the past ten years, and related applications can be found in various areas, e.g., distributed optimization, robot/vehicle formation and social networks [1][9]. A fundamental control problem of MASs is consensus, that is, to find a control protocol such that the states of all agents converge to some common trajectory. A basic requirement about a control protocol for MASs is the fact that it can be implemented in a distributed way. This is because agents in practice usually have limited communication capabilities such that each agent can only communicate with its neighbours. Up to now, there have been many results that can manage this challenge. To mention a few, consensus seeking of singleintegrator systems was investigated in [10], where it was shown that a directed spanning tree is necessary for consensus

This work was supported by the Alexander von Humboldt Foundation of Germany and by the joint Sino-German project "Control and Optimization for Event-triggered Networked Autonomous Multi-agent Systems" funded by the German Research Foundation (DFG) and the National Science Foundation of China (NSFC).

$\mathrm{X}$. Li is with the Department of Automation, Shanghai Jiao Tong University, Shanghai 200240, China (e-mail: lixianwei1985@gmail.com).

F. Liu and M. Buss are with Chair of Automatic Control Engineering, Technical University of Munich, 80333 Munich, Germany (e-mail: fangzhou.liu@tum.de; mb@tum.de).

S. Hirche is with Chair of Information-oriented Control, Technical University of Munich, 80333 Munich, Germany (e-mail: hirche@tum.de). of MASs; consensus of general linear MASs was studied in [11], and a kind of dynamic output-feedback protocols with controller interaction were proposed; in [12], [13], a novel dynamic output-feedback protocol was proposed for general linear MASs, and moreover, a unified robust control point of view was provided for the existence of output-feedback protocols without exchanging controller information.

A drawback of the aforementioned results is the fact that the smallest nonzero eigenvalue of the Laplacian matrix is needed for protocol design. This requires that the weights of the communication graph must be exactly known. Moreover, even if the graph is known, it is still difficult to exactly compute the eigenvalues of the Laplacian matrix when the network size is large. As a result, although the protocols in the aforementioned results can be implemented distributively, the corresponding design process is not of the distributed nature. Inspired by this limitation, many efforts have been made to explore fully distributed control protocols for consensus of linear MASs, especially those adaptively adjusting scalar gains about the graph by using local information only [14]-[18]. Fully distributed state-feedback protocols were constructed for general linear MASs in [14], [16] with undirected and directed graphs, respectively. Adaptive consensus of linear MASs with external disturbances are further studied under a kind of dynamic state-feedback protocols in [18]. Inspired by the output-feedback protocols in [11], adaptive dynamic output-feedback protocols were proposed in [15] for general linear MASs with undirected graphs, where leader-follower consensus with the leader having an nonzero input was also discussed. To deal with directed graphs, the authors further proposed a kind of sequential observer based adaptive outputfeedback protocols in [17] for linear MASs.

Although there have been some results about fully distributed consensus control of MASs as mentioned above, how to reduce the complexity of the constructed protocols is still challenging. Particularly, the order of each local controller of the protocols in [15] is identical to that of the agents. In view of this fact, we term a protocol like those in [15] as a full-order protocol. As such, it is seen that the dynamic output-feedback protocols without the adaptive mechanism in [11], [12], [19] also fall into the full-order type. Based on this observation, a natural idea for reducing the protocol complexity is to design reduced-order protocols, that is, the order of each local controller is smaller than that of the 
agents; see [20], [21]. However, note that no fully distributed reduced-order protocol has been developed for MASs. The reduced-order protocols in [20], [21] are based on the design theory of traditional reduced-order observer-based controllers. In addition to solving some matrix equations, they also need to know the smallest nonzero eigenvalue of the Laplacian matrix. By using local output information only, it is unknown yet whether the protocols in [20], [21] can be extended to the case that the graph related gains are adaptively adjusted. Moreover, note that the protocols therein have their own shortcomings. Specifically, the one in [20] requires absolute output information of individual agents, which is impractical in some applications; while the one in [21] requires relative input information between neighbouring agents, such that the underlying graph over which the controller information flows is actually different from the original communication graph. It is thus open and challenging to design fully distributed reducedorder output-feedback protocols for consensus of MASs.

Motivated by the above observations, this paper investigates the fully distributed consensus control problem of linear MASs via reduced-order adaptive output-feedback control protocols. The communication graph is assumed to be undirected. Two kind of reduced-order adaptive dynamic output-feedback protocols are constructed: the first one updates the scalar gains from the output difference of the two agents on each edge, termed an edge-based protocol, while the second one from the output difference sum of all neighbouring agents for each node, termed a node-based protocol. By appropriately parameterizing the protocol gains, existence conditions are derived for the two protocols, which require the solvability of some matrix equations related to agent dynamics. Moreover, a design algorithm is presented, which shows that the proposed adaptive output-feedback protocols with $\left(n_{x}-n_{u}\right)$ thorder local controllers are always feasible, provided that the agents are stabilizable and detectable and the communication graph is connected. Thus, like the full-order results in [15], the proposed results confirm that some reduced-order fully distributed consensus protocols must exist and can be easily found. Moreover, compared with the reduced-order protocols in [20], [21], the proposed protocols make use of relative output information more straightforwardly. The efficacy of the proposed theoretical results are finally demonstrated by a simulation example about formation flying of spacecrafts.

Notation: Represent the set of all $m \times n$ real matrices by $\mathbb{R}^{m \times n}$ and an $n \times n$ identity matrix by $\mathbf{I}_{n}$, where the subscript is omitted if no confusion is caused. A square, positive definite (semi-definite) matrix is denoted by $P>0(\geq 0)$. Kronecker product and Hadamard product for two matrices $A$ and $B$ are represented by $A \otimes B$ and $A \circ B$, respectively. For (block) diagonal matrix with $A_{1}, \ldots, A_{n}$ on the diagonal, we write it as $\operatorname{diag}\left\{A_{1}, \ldots, A_{n}\right\}$. Image $(\cdot)$ indicates the image of a matrix $(\cdot) .\|(\cdot)\|$ denotes the 2 -norm of a vector $(\cdot)$.

An undirected graph is denoted by $\mathcal{G}(\mathcal{V}, \mathcal{E})$ with $\mathcal{V}=$ $\{1, \ldots, N\}$ the set of $N$ nodes and $\mathcal{E} \subseteq \mathcal{V} \times \mathcal{V}$ the edge set. The associated adjacency matrix is denoted by $\mathcal{A}=\left[a_{i j}\right]_{N \times N}$, where $a_{i j}>0$ if $(j, i) \in \mathcal{E}$ and $a_{i j}=0$ otherwise. For an undirected graph, we mean $a_{i j}=a_{j i}$ for $i, j=1, \ldots, N$. If $(j, i) \in \mathcal{E}$ or $a_{i j}>0$, node $j$ is said to be a neighbouring node of node $i$ and the set of all the neighbouring node of node $i$ is denoted by $\mathcal{N}_{i}$. The associated Laplacian matrix $\mathcal{L}=\left[l_{i j}\right]_{N \times N}$ is defined as $l_{i i}=\sum_{k \in \mathcal{N}_{i}} a_{i k}$ and $l_{i j}=-a_{i j}$ for $i, j=1,2, \ldots, N$ and $i \neq j$. A path of the graph is a sequence of edges connecting two nodes. We say an undirected graph is connected if every node can be reached from every other node over any path.

Lemma 1 ([22, Lemma 1]): Consider an undirected graph $\mathcal{G}(\mathcal{V}, \mathcal{E})$ and suppose it is connected. Then zero is a simple eigenvalue of the Laplacian matrix $\mathcal{L}$, and $\lambda_{2}(\mathcal{L})=$ $\min _{1^{\mathrm{T}} x=0, x \neq 0} \frac{x^{\mathrm{T}} \mathcal{L} x}{x^{\mathrm{T}} x}>0$ is the smallest nonzero eigenvalue.

\section{MAin Results}

We will present two reduced-order adaptive output-feedback protocols for consensus control of general linear MASs. The consensus problem will be first formulated. Then consensus analysis conditions for the concerned protocols will be provided, and finally a design algorithm will be presented.

\section{A. Problem Statement}

Consider $N(N \geq 2)$ homogeneous dynamic agents with each one represented by a linear time-invariant system:

$$
\begin{aligned}
\dot{x}_{i}(t) & =A x_{i}(t)+B_{u} u_{i}(t), \\
y_{i}(t) & =C_{y} x_{i}(t), i=1, \ldots, N,
\end{aligned}
$$

where $x_{i} \in \mathbb{R}^{n_{x}}, u_{i} \in \mathbb{R}^{n_{u}}$ and $y_{i} \in \mathbb{R}^{n_{y}}$ are the state, control input and local output of agent $i$, respectively, and $A, B_{u}$ and $C_{y}$ are appropriately-dimensioned real system matrices. Without loss of generality, we assume that the matrix triple $\left(A, B_{u}, C_{y}\right)$ is stabilizable and detectable.

The consensus problem is to find a control protocol that drives the states of all the agents to track common trajectories. Denote by an undirected graph $\mathcal{G}(\mathcal{V}, \mathcal{E})$ the communication topology of a distributed protocol. We are interested in distributed control protocols that make only use of relative information between neighbouring agents. Symbols with accent " $\sim$ " are used to denote the relative information between neighbouring agents. For instance,

$$
\begin{aligned}
& \tilde{x}_{i}(t) \triangleq \sum_{j \in \mathcal{N}_{i}} a_{i j}\left(x_{i}(t)-x_{j}(t)\right)=\sum_{j=1}^{N} l_{i j} x_{j}(t), \\
& \tilde{y}_{i}(t) \triangleq \sum_{j \in \mathcal{N}_{i}} a_{i j}\left(y_{i}(t)-y_{j}(t)\right)=\sum_{j=1}^{N} l_{i j} y_{j}(t),
\end{aligned}
$$

where $\mathcal{A}=\left[a_{i j}\right]_{N \times N}$ and $\mathcal{L}=\left[l_{i j}\right]_{N \times N}$ are the adjacency matrix and Laplacian matrix of $\mathcal{G}(\mathcal{V}, \mathcal{E})$, respectively, and $\mathcal{N}_{i}$ is the set of neighbouring agents to agent $i$. Signals $\tilde{x}_{i}$ and $\tilde{y}_{i}$ are the relative state and relative output of agent $i$, respectively. Protocols making use of $\tilde{x}_{i}$ for consensus control are called state-feedback protocols, while those making use of $\tilde{y}_{i}$ are output-feedback protocols. Since full (relative) state information is not always available, output-feedback protocols are of more relevance in applications.

To make full use of relative output $\tilde{y}_{i}$, we propose two distributed adaptive output-feedback protocols for consensus 
control. The edge-based output-feedback protocol is given by

$$
\begin{aligned}
& \dot{r}_{i}(t)=H r_{i}(t)+F_{r} \sum_{j \in \mathcal{N}_{i}} c_{i j}(t) a_{i j}\left(\tilde{y}_{i j}(t)+C_{y} B_{r} \tilde{r}_{i j}(t)\right), \\
& u_{i}(t)=G r_{i}(t)+F_{u} \sum_{j \in \mathcal{N}_{i}} c_{i j}(t) a_{i j}\left(\tilde{y}_{i j}(t)+C_{y} B_{r} \tilde{r}_{i j}(t)\right),
\end{aligned}
$$

with the law of adaption

$$
\begin{gathered}
\dot{c}_{i j}(t)=\alpha_{i j}\left(\tilde{y}_{i j}(t)+C_{y} B_{r} \tilde{r}_{i j}(t)\right)^{\mathrm{T}}\left(\tilde{y}_{i j}(t)+C_{y} B_{r} \tilde{r}_{i j}(t)\right), \\
c_{i j}(0)=c_{j i}(0)>0, \alpha_{i j}=\alpha_{j i}, i=1, \ldots, N ; j \in \mathcal{N}_{i}, \quad(4)
\end{gathered}
$$

while the node-based output-feedback protocol is given by

$$
\begin{aligned}
& \dot{r}_{i}(t)=H r_{i}(t)+d_{i}(t) F_{r}\left(\tilde{y}_{i}(t)+C_{y} B_{r} \tilde{r}_{i}(t)\right), \\
& u_{i}(t)=G r_{i}(t)+d_{i}(t) F_{u}\left(\tilde{y}_{i}(t)+C_{y} B_{r} \tilde{r}_{i}(t)\right),
\end{aligned}
$$

with the law of adaption

$$
\begin{aligned}
\dot{d}_{i}(t) & =\beta_{i}\left(\tilde{y}_{i}(t)+C_{y} B_{r} \tilde{r}_{i}(t)\right)^{\mathrm{T}}\left(\tilde{y}_{i}(t)+C_{y} B_{r} \tilde{r}_{i}(t)\right), \\
d_{i}(0) & >0, i=1, \ldots, N .
\end{aligned}
$$

Here, $\alpha_{i j}$ and $\beta_{i}$ are any positive scalar constants and $r_{i} \in \mathbb{R}^{n_{r}}$ is the local controller state for agent $i$ and

$$
\begin{aligned}
\tilde{y}_{i j}(t) & \triangleq y_{i}(t)-y_{j}(t), \tilde{r}_{i j}(t) \triangleq r_{i}(t)-r_{j}(t), \\
\tilde{r}_{i}(t) & \triangleq \sum_{j \in \mathcal{N}_{i}} a_{i j}\left(r_{i}(t)-r_{j}(t)\right)=\sum_{j=1}^{N} l_{i j} r_{j}(t) .
\end{aligned}
$$

Matrix gains $H, G, B_{r}, F_{r}$ and $F_{u}$, which have proper dimensions, are protocol parameters to be designed. Note that the order of the local controller, $n_{r}$, is not required to be such that $n_{r}=n_{x}$, that is, local controllers are not required to be of full order as that of agents. A kind of adaptive fullorder dynamic output-feedback protocols have been addressed in [15]. However, it will be clear that this paper can deal with both full-order and reduced-order cases. Following [15], the two notions, edge-based protocols and node-based protocols are due to the information used for computing the adaptive gains. Adaptive gains are associated with each edge for the former case, and with each node for the latter case.

What is worth pointing out is that our goal is to provide a tractable characterization of reduced-order adaptive outputfeedback protocols. On one hand, although the above protocols seem to have a special form, it will be seen in Section II-D that the proposed design method provides a more general formulation of some existing results. On the other hand, it is known that designing a reduced-order output-feedback controller is not tractable in general. Without a proper parameterization of the gain matrices, even if one can formulate a general linear dynamic output-feedback protocol (e.g., a node-based protocol like $\dot{r}_{i}=A_{r} r_{i}+B_{r} \tilde{r}_{i}+B_{y} \tilde{y}_{i}$ and $\left.u_{i}=C_{r} r_{i}+D_{r} \tilde{r}_{i}+D_{y} \tilde{y}_{i}\right)$, finding feasible gain matrices is still not tractable.

The consensus control problem to be addressed in this paper is stated as follows: For the MAS (1), find an output-feedback protocol (3) (resp., (5)) such that the states of the resulting closed-loop system satisfy $\lim _{t \rightarrow \infty}\left\|x_{i}(t) \rightarrow x_{j}(t)\right\|=0$ and $\lim _{t \rightarrow \infty}\left\|r_{i}(t) \rightarrow r_{j}(t)\right\|=0$ for all $i, j=1, \ldots, N$.

Remark 1: Although the protocols (3) and (5) are motivated by combining adaptive designs and reduced-order designs, a main challenge of designing reduced-order adaptive protocols is how to establish a proper law of adaption on some "error" while keeping the tractability of design conditions. For instance, the law in $[15,(2)]$ is given by $\dot{c}_{i j}=\varepsilon_{i j} a_{i j}\left(\tilde{y}_{i j}-C_{y} \tilde{r}_{i j}\right)^{\mathrm{T}}\left(\tilde{y}_{i j}-C_{y} \tilde{r}_{i j}\right)$, where $\tilde{y}_{i j}-C_{y} \tilde{r}_{i j}$ might be intuitively understood as the "error". However, it is unclear what such an "error" should be for the existing reduced-order protocols in [20], [21]. For the protocols (3) and (5), since $r_{i}$ are some intermediate variables for control but of no physical meaning, it is also difficult to tell what such an "error" should be without carefully designing the laws (4) and (6), let alone, as explained before, the fact that different parameterizations are adopted in (3)/(5) and [20], [21].

Remark 2: On one hand, the first difference between the two protocols is obviously the number of adaptive gains. Note that the smallest number of edges for a connected undirected graph is $N-1$. In such an extreme case, the number of adaptive gains for (3) is $2(N-1)$. Thus, for any connected undirected graph with $N \geq 3$, the number of adaptive gains for (3) is always larger than that for (5). In other words, the edgebased protocol (3) in general involves more adaptive gains than the node-based one (5), which implies that (3) is more complex. On the other hand, if we see $c_{i j} a_{i j}$ and $d_{i} a_{i j}$ as the time-varying weights of edge $(i, j)$ of the new communication graph, then the Laplacian of the new graph for the former case keeps symmetric while that for the latter does not. This symmetry might benefit system analysis. For instance, if the adaptive gains are fixed after converging, the communication graph of the closed-loop system is undirected with a symmetric Laplacian, to which many existing results for MASs on fixed undirected graphs can be applied.

Remark 3: The adaptive gains $c_{i j}$ and $d_{i}$ are non-decreasing when consensus has not been precisely reached. Thus, if the system is subject to external disturbances such that the consensus error is not convergent to zero, then $c_{i j}$ and $d_{i}$ could continuous increasing to infinity. To circumvent this drawback, one may use the so-called $\sigma$-modification technique to damp the adaptive gains. Nevertheless, a trade-off that in general has to be made is no convergence guarantee, but only boundedness guarantee, for the consensus error, if the external disturbances are assumed to be bounded (see [15], [23]).

\section{B. Consensus Analysis Under the Edge-Based Protocol}

In this subsection, we present some sufficient conditions under which the closed-loop system resulting from the edgebased protocol (3) reaches consensus. The case for the nodebased one (5) will be discussed in the next subsection.

1) Error Dynamics: Let $c_{i i}=\frac{\sum_{j \in \mathcal{N}_{i}} c_{i j} a_{i j}}{l_{i i}}$ for $i=$ $1, \ldots, N$ and $c_{i j}=0$ for $i=1, \ldots, N$ and $j \notin \mathcal{N}_{i}$. Thus,

$$
\begin{aligned}
& \sum_{j \in \mathcal{N}_{i}} c_{i j}(t) a_{i j}\left(\tilde{y}_{i j}(t)+C_{y} B_{r} \tilde{r}_{i j}(t)\right) \\
& =\sum_{j=1}^{N} c_{i j}(t) l_{i j}\left(y_{j}(t)+C_{y} B_{r} r_{j}(t)\right) .
\end{aligned}
$$

Define the state vector $s \triangleq \operatorname{col}\left\{s_{1}, \ldots, s_{N}\right\}$ with $s_{i} \triangleq$ $\operatorname{col}\left\{x_{i}, r_{i}\right\}$. By combining the agents with the protocols (3), 
the closed-loop system is given by

$$
\dot{s}(t)=[\mathbf{I} \otimes \tilde{A}+(\mathcal{C}(t) \circ \mathcal{L}) \otimes \tilde{B}] s(t),
$$

where $\mathcal{C} \triangleq\left[c_{i j}\right]_{N \times N}$ and

$$
\tilde{A} \triangleq\left[\begin{array}{cc}
A & B_{u} G \\
0 & H
\end{array}\right], \tilde{B} \triangleq\left[\begin{array}{cc}
B_{u} F_{u} C_{y} & B_{u} F_{u} C_{y} B_{r} \\
F_{r} C_{y} & F_{r} C_{y} B_{r}
\end{array}\right] .
$$

Furthermore, define the state transformation $s_{e} \triangleq\left(\mathcal{L}_{e} \otimes \mathbf{I}\right) s$, where $\mathcal{L}_{e} \triangleq \mathbf{I}-\frac{1}{N} \mathbf{1 1}^{\mathrm{T}}$. Denote $s_{e}=\operatorname{col}\left\{s_{e 1}, \ldots, s_{e N}\right\}$ and thus $s_{e i}=s_{i}-\frac{1}{N} \sum_{j=1}^{N} s_{j}$. Since $c_{i j}(0)=c_{j i}(0)$ and $\alpha_{i j}=$ $\alpha_{j i}$ imply $c_{i j}(t)=c_{j i}(t)$ for all $t \geq 0$ and $\mathcal{L}$ is symmetric, we have $\mathcal{L}_{e}(\mathcal{C}(t) \circ \mathcal{L})=\mathcal{C}(t) \circ \mathcal{L}=(\mathcal{C}(t) \circ \mathcal{L}) \mathcal{L}_{e}$. Thus, the closed-loop system (7) can be transformed into the following form:

$$
\begin{aligned}
\dot{s}_{e}(t) & =\left(\mathcal{L}_{e} \otimes \mathbf{I}\right)[\mathbf{I} \otimes \tilde{A}+(\mathcal{C}(t) \circ \mathcal{L}) \otimes \tilde{B}] s(t) \\
& =[\mathbf{I} \otimes \tilde{A}+(\mathcal{C}(t) \circ \mathcal{L}) \otimes \tilde{B}] s_{e}(t),
\end{aligned}
$$

The following lemma can be obtained, which bridges the consensus property of the original MAS and the convergence property of the system (8) under the protocol (3).

Lemma 2: For the MAS (1) with the protocol (3), consensus is reached if and only if $s_{e}(t) \rightarrow 0$ as $t \rightarrow \infty$.

Proof: Note that $\mathcal{L}_{e} q=0$ for some vector $q$ if and only if $q \in$ Image $(\mathbf{1})$. Thus, $s_{e}(t)=\left(\mathcal{L}_{e} \otimes \mathbf{I}\right) s(t) \rightarrow 0$ as $t \rightarrow \infty$ if and only if $s(t) \rightarrow \operatorname{Image}(\mathbf{1} \otimes \mathbf{I})$ as $t \rightarrow \infty$, which is obviously equivalent to the fact that consensus is reached.

2) Existence Condition: Now we present the following result on the existence of a distributed adaptive output-feedback protocol (3) such that consensus is reached.

Theorem 1: Consider the MAS (1) and the protocol (3), and suppose that the communication graph $\mathcal{G}$ is undirected and connected. Then consensus is reached, and $c_{i j}(t), i, j=$ $1, \ldots, N$, converge to some finite positive constants as $t \rightarrow \infty$, if the following statements hold:

1) Matrices $H, G$ and $B_{r}$ are such that $H$ is Hurwitz and

$$
A B_{r}-B_{r} H=B_{u} G \text {. }
$$

2) Matrices $F_{u}$ and $F_{r}$ are such that

$$
B_{u} F_{u}+B_{r} F_{r}=-P C_{y}^{\mathrm{T}}
$$

where $P$ is a positive definite matrix solving the following ARE for any positive definite matrix $Q$ :

$$
P A^{\mathrm{T}}+A P-P C_{y}^{\mathrm{T}} C_{y} P+Q=0
$$

Proof: Introduce the following matrix $T$ and its inverse $T^{-1}$, which are obviously well-defined:

$$
T=\left[\begin{array}{cc}
\mathbf{I}_{n_{x}} & B_{r} \\
0 & \mathbf{I}_{n_{r}}
\end{array}\right], T^{-1}=\left[\begin{array}{cc}
\mathbf{I}_{n_{x}} & -B_{r} \\
0 & \mathbf{I}_{n_{r}}
\end{array}\right] .
$$

By substituting the equations (9) and (10), we obtain

$$
\begin{aligned}
\bar{A} & \triangleq T \tilde{A} T^{-1}=\left[\begin{array}{cc}
\mathbf{I}_{n_{x}} & B_{r} \\
0 & \mathbf{I}_{n_{r}}
\end{array}\right]\left[\begin{array}{cc}
A & B_{u} G \\
0 & H
\end{array}\right]\left[\begin{array}{cc}
\mathbf{I}_{n_{x}} & -B_{r} \\
0 & \mathbf{I}_{n_{r}}
\end{array}\right] \\
& =\left[\begin{array}{cc}
A & -A B_{r}+B_{u} G+B_{r} H \\
0 & H
\end{array}\right]=\left[\begin{array}{cc}
A & 0 \\
0 & H
\end{array}\right],
\end{aligned}
$$

$\bar{B} \triangleq T \tilde{B} T^{-1}$

$$
\begin{aligned}
= & {\left[\begin{array}{cc}
\mathbf{I}_{n_{x}} & B_{r} \\
0 & \mathbf{I}_{n_{r}}
\end{array}\right]\left[\begin{array}{cc}
B_{u} F_{u} C_{y} & B_{u} F_{u} C_{y} B_{r} \\
F_{r} C_{y} & F_{r} C_{y} B_{r}
\end{array}\right] } \\
& \times\left[\begin{array}{cc}
\mathbf{I}_{n_{x}} & -B_{r} \\
0 & \mathbf{I}_{n_{r}}
\end{array}\right] \\
= & {\left[\begin{array}{cc}
B_{u} F_{u} C_{y}+B_{r} F_{r} C_{y} & 0 \\
F_{r} C_{y} & 0
\end{array}\right]=\left[\begin{array}{cc}
-P C_{y}^{\mathrm{T}} C_{y} & 0 \\
F_{r} C_{y} & 0
\end{array}\right] . }
\end{aligned}
$$

Perform a state transformation $\bar{s}=\operatorname{col}\left\{\bar{s}_{1}, \ldots, \bar{s}_{N}\right\} \triangleq$ $(\mathbf{I} \otimes T) s_{e}$. Then it follows from (8) that

$$
\begin{aligned}
\dot{\bar{s}}(t) & =(\mathbf{I} \otimes T)[\mathbf{I} \otimes \tilde{A}+(\mathcal{C}(t) \circ \mathcal{L}) \otimes \tilde{B}] s_{e}(t) \\
& =[\mathbf{I} \otimes \bar{A}+(\mathcal{C}(t) \circ \mathcal{L}) \otimes \bar{B}] \bar{s}(t) .
\end{aligned}
$$

From Lemma 2, it is known that consensus is reached if and only if $s_{e}(t) \rightarrow 0$ as $t \rightarrow \infty$. Since the state transformation from $s_{e}$ to $\bar{s}$ is invertible, requiring $s_{e}(t) \rightarrow 0$ as $t \rightarrow \infty$ is equivalent to requiring $\bar{s}(t) \rightarrow 0$ as $t \rightarrow \infty$. Alternatively, the state equations of $\bar{s}(t)$ as above can be represented by

$$
\begin{aligned}
\dot{\bar{x}}(t) & =\left[\mathbf{I} \otimes A-(\mathcal{C}(t) \circ \mathcal{L}) \otimes P C_{y}^{\mathrm{T}} C_{y}\right] \bar{x}(t), \\
\dot{\bar{r}}(t) & =(\mathbf{I} \otimes H) \bar{r}(t)+\left[(\mathcal{C}(t) \circ \mathcal{L}) \otimes F_{r} C_{y}\right] \bar{x}(t),
\end{aligned}
$$

where

$$
\left[\begin{array}{c}
\bar{x} \\
\bar{r}
\end{array}\right]=\left[\begin{array}{c}
\operatorname{col}\left\{\bar{x}_{1}, \ldots, \bar{x}_{N}\right\} \\
\operatorname{col}\left\{\bar{r}_{1}, \ldots, \bar{r}_{N}\right\}
\end{array}\right]=\left[\begin{array}{l}
\mathbf{I} \otimes\left[\mathbf{I}_{n_{x}}, 0_{n_{x} \times n_{r}}\right] \\
\mathbf{I} \otimes\left[0_{n_{r} \times n_{x}}, \mathbf{I}_{n_{r}}\right]
\end{array}\right] \bar{s}
$$

Obviously, consensus is reached if and only if $\bar{x}(t) \rightarrow 0$ and $\bar{r}(t) \rightarrow 0$ as $t \rightarrow \infty$. Note that $\bar{x}$ is not affected by Moreover, $\bar{r}$ is governed by a linear time-invariant system with $\bar{x}$ as the input and this system is asymptotically stable since $H$ is assumed to be Hurwitz. Thus, hereafter we only need to prove that $\mathcal{C}(t)$ is bounded and $\bar{x}(t) \rightarrow 0$ as $t \rightarrow \infty$.

For the system (13), construct a candidate Lyapunov function as

$V(t)=\bar{x}^{\mathrm{T}}(t)\left(\mathbf{I} \otimes P^{-1}\right) \bar{x}(t)+\sum_{i=1}^{N} \sum_{j=1, j \neq i}^{N} \frac{a_{i j}}{2 \alpha_{i j}}\left(c_{i j}(t)-\bar{c}\right)^{2}$,

where $\bar{c}$ is a positive constant to be determined and $P$ is the positive definite matrix given in Statement 2 of the theorem. Taking the derivative of $V(t)$ along the solution of $\bar{x}(t)$ in (13) and $c_{i j}(t)$ in (4), we have

$$
\begin{aligned}
\dot{V}= & 2 \bar{x}^{\mathrm{T}}\left(\mathbf{I} \otimes P^{-1}\right) \dot{\bar{x}}+\sum_{i=1}^{N} \sum_{j=1, j \neq i}^{N} \frac{a_{i j}}{\alpha_{i j}}\left(c_{i j}-\bar{c}\right) \dot{c}_{i j} \\
= & 2 \bar{x}^{\mathrm{T}}\left(\mathbf{I} \otimes P^{-1}\right)\left[\mathbf{I} \otimes A-(\mathcal{C} \circ \mathcal{L}) \otimes P C_{y}^{\mathrm{T}} C_{y}\right] \bar{x} \\
& +\sum_{i=1}^{N} \sum_{j=1, j \neq i}^{N}\left(a_{i j} c_{i j}-a_{i j} \bar{c}\right)\left(\tilde{y}_{i j}+C_{y} B_{r} \tilde{r}_{i j}\right)^{\mathrm{T}} \\
& \times\left(\tilde{y}_{i j}+C_{y} B_{r} \tilde{r}_{i j}\right) .
\end{aligned}
$$

Since

$$
\begin{aligned}
\tilde{y}_{i j}+C_{y} B_{r} \tilde{r}_{i j} & =C_{y}\left[\begin{array}{ll}
\mathbf{I} & B_{r}
\end{array}\right]\left(s_{i}-s_{j}\right) \\
& =C_{y}\left[\begin{array}{ll}
\mathbf{I} & B_{r}
\end{array}\right]\left(s_{e i}-s_{e j}\right) \\
& =C_{y}\left[\begin{array}{ll}
\mathbf{I} & B_{r}
\end{array}\right] T^{-1}\left(\bar{s}_{i}-\bar{s}_{j}\right) \\
& =C_{y}\left[\begin{array}{ll}
\mathbf{I} & B_{r}
\end{array}\right]\left[\begin{array}{cc}
\mathbf{I}_{n_{x}} & -B_{r} \\
0 & \mathbf{I}_{n_{r}}
\end{array}\right]\left(\bar{s}_{i}-\bar{s}_{j}\right)
\end{aligned}
$$




$$
=C_{y}\left[\begin{array}{ll}
\mathbf{I} & 0
\end{array}\right]\left(\bar{s}_{i}-\bar{s}_{j}\right)=C_{y}\left(\bar{x}_{i}-\bar{x}_{j}\right)
$$

and $c_{i j}(t)=c_{j i}(t)$ for all $t \geq 0$, we have

$$
\begin{aligned}
& \sum_{i=1}^{N} \sum_{j=1, j \neq i}^{N}\left(a_{i j} c_{i j}-a_{i j} \bar{c}\right)\left(\tilde{y}_{i j}+C_{y} B_{r} \tilde{r}_{i j}\right)^{\mathrm{T}}\left(\tilde{y}_{i j}+C_{y} B_{r} \tilde{r}_{i j}\right) \\
& =\sum_{i=1}^{N} \sum_{j=1, j \neq i}^{N}\left(a_{i j} c_{i j}-a_{i j} \bar{c}\right)\left(\bar{x}_{i}-\bar{x}_{j}\right)^{\mathrm{T}} C_{y}^{\mathrm{T}} C_{y}\left(\bar{x}_{i}-\bar{x}_{j}\right) \\
& =2 \sum_{i=1}^{N} \sum_{j=1, j \neq i}^{N}\left(a_{i j} c_{i j}-a_{i j} \bar{c}\right) \bar{x}_{i}^{\mathrm{T}} C_{y}^{\mathrm{T}} C_{y}\left(\bar{x}_{i}-\bar{x}_{j}\right) \\
& =2 \bar{x}^{\mathrm{T}}\left[(\mathcal{C} \circ \mathcal{L}-\bar{c} \mathcal{L}) \otimes C_{y}^{\mathrm{T}} C_{y}\right] \bar{x} .
\end{aligned}
$$

Substituting this equation into $\dot{V}(t)$ leads to

$$
\dot{V}=\bar{x}^{\mathrm{T}}\left[\mathbf{I} \otimes\left(P^{-1} A+A^{\mathrm{T}} P^{-1}\right)\right] \bar{x}-2 \bar{c} \bar{x}^{\mathrm{T}}\left(\mathcal{L} \otimes C_{y}^{\mathrm{T}} C_{y}\right) \bar{x} .
$$

Since $\left(\mathbf{1}^{\mathrm{T}} \otimes \mathbf{I}\right)\left(\mathbf{I} \otimes C_{y}\right) \bar{x}=\left(\mathbf{1}^{\mathrm{T}} \mathcal{L}_{e} \otimes C_{y}[\mathbf{I}, 0] T\right) s=0$, it follows from Lemma 1 that

$$
\bar{x}^{\mathrm{T}}\left(\mathcal{L} \otimes C_{y}^{\mathrm{T}} C_{y}\right) \bar{x} \geq \lambda_{2}(\mathcal{L}) \bar{x}^{\mathrm{T}}\left(\mathbf{I} \otimes C_{y}^{\mathrm{T}} C_{y}\right) \bar{x} .
$$

Let $\bar{c}$ be any constant such that $\bar{c} \geq \frac{1}{2} \lambda_{2}^{-1}(\mathcal{L})$. Using these relations results in

$$
\begin{aligned}
\dot{V} & \leq \bar{x}^{\mathrm{T}}\left[\mathbf{I} \otimes\left(P^{-1} A+A^{\mathrm{T}} P^{-1}\right)\right] \bar{x}-2 \bar{c} \lambda_{2}(\mathcal{L}) \bar{x}^{\mathrm{T}}\left(\mathbf{I} \otimes C_{y}^{\mathrm{T}} C_{y}\right) \bar{x} \\
& \leq \bar{x}^{\mathrm{T}}\left[\mathbf{I} \otimes\left(P^{-1} A+A^{\mathrm{T}} P^{-1}\right)\right] \bar{x}-\bar{x}^{\mathrm{T}}\left(\mathbf{I} \otimes C_{y}^{\mathrm{T}} C_{y}\right) \bar{x} \\
& =\bar{x}^{\mathrm{T}}\left(\mathbf{I} \otimes P^{-1} Q P^{-1}\right) \bar{x} \leq 0 .
\end{aligned}
$$

Since $\dot{V}(t) \leq 0$ and $V(t) \geq 0$ for all $t \geq 0$, it is seen that $V(t)$ is bounded, which implies that $c_{i j}(t)$ and thus $\mathcal{C}(t)$ are bounded. Moreover, since $\dot{c}_{i j}(t) \geqq 0$ and $c_{i j}(t)>0$, it is proved that $c_{i j}(t)$ converge to some finite positive constants. In addition, because $\dot{V}(t) \leq 0$ and $\dot{V}(t)=0$ implies $\bar{x}(t)=0$, it is known from Lasalle's theorem (see [24, Theorem 4.4]) that $\bar{x}(t) \rightarrow 0$ as $t \rightarrow \infty$. Consequently, consensus is reached under the protocol (3). The proof is completed.

\section{Consensus Analysis Under the Node-Based Protocol}

In this subsection, the consensus property of the closedloop system under the node-based protocol (5) will be analysed. Define $\mathcal{D} \triangleq \operatorname{diag}\left\{d_{1}, \ldots, d_{N}\right\}$. Then the corresponding closed-loop system is given by

$$
\dot{s}(t)=(\mathbf{I} \otimes \tilde{A}+\mathcal{D}(t) \mathcal{L} \otimes \tilde{B}) s(t) .
$$

Note that $\mathcal{D}(t) \mathcal{L} \mathcal{L}_{e}=\mathcal{D}(t) \mathcal{L}$. Thus $s_{e}(t)$ satisfies

$$
\dot{s}_{e}(t)=\left(\mathbf{I} \otimes \tilde{A}+\mathcal{L}_{e} \mathcal{D}(t) \mathcal{L} \otimes \tilde{B}\right) s_{e}(t) .
$$

Similar to Lemma 2, we can establish the equivalence of the consensus of the original system and the convergence of $s_{e}(t)$. As a result, we can obtain the following consensus condition through proving the convergence of $s_{e}(t)$ in (15).

Theorem 2: Consider the MAS (1) and the protocol (5), and suppose that the communication graph $\mathcal{G}$ is undirected and connected. Then consensus is reached, and $d_{i}(t), i=$ $1, \ldots, N$, converge to some finite positive constants as $t \rightarrow \infty$, if Statements 1) and 2) in Theorem 1 hold. satisfies

Proof: Similar to (12), the new state $\bar{s}(t)=(\mathbf{I} \otimes T) s_{e}$

$$
\dot{\bar{s}}(t)=\left(\mathbf{I} \otimes \bar{A}+\mathcal{L}_{e} \mathcal{D}(t) \mathcal{L} \otimes \bar{B}\right) \bar{s}(t) .
$$

Moreover, similar to (13), the above system can be written as

$$
\begin{aligned}
\dot{\bar{x}}(t) & =\left(\mathbf{I} \otimes A-\mathcal{L}_{e} \mathcal{D}(t) \mathcal{L} \otimes P C_{y}^{\mathrm{T}} C_{y}\right) \bar{x}(t), \\
\dot{\bar{r}}(t) & =(\mathbf{I} \otimes H) \bar{r}(t)+\left(\mathcal{L}_{e} \mathcal{D}(t) \mathcal{L} \otimes F_{r} C_{y}\right) \bar{x}(t) .
\end{aligned}
$$

Since the second subsystem is stable, to prove the convergence of $\bar{s}_{e}(t)$ through $\bar{s}(t)$, we only need to prove the convergence of $\bar{x}(t)$. Construct a candidate Lyapunov function as

$$
W(t)=\bar{x}^{\mathrm{T}}(t)\left(\mathcal{L} \otimes P^{-1}\right) \bar{x}(t)+\sum_{i=1}^{N} \frac{1}{\beta_{i}}\left(d_{i}(t)-\bar{d}\right)^{2},
$$

where $\bar{d}$ is a positive constant to be determined and $P$ is the positive definite matrix satisfying (11). Since the communication graph is assumed to be undirected and connected and there holds $\left(\mathbf{1}^{\mathrm{T}} \otimes \mathbf{I}\right) \bar{x}=\left(\mathbf{1}^{\mathrm{T}} \mathcal{L}_{e} \otimes[\mathbf{I}, 0] T\right) s=0$, it follows from Lemma 1 that $\bar{x}^{\mathrm{T}}\left(\mathcal{L} \otimes P^{-1}\right) \bar{x} \geq \lambda_{2}(\mathcal{L}) \bar{x}^{\mathrm{T}}\left(\mathbf{I} \otimes P^{-1}\right) \bar{x} \geq 0$. Thus, $W \geq 0$ and the equality holds only if $\bar{x}=0$. Taking the derivative of $W$ along the solution of $\bar{x}$ in (16) and $d_{i}$ in (6), we have

$$
\begin{aligned}
\dot{W}= & 2 \bar{x}^{\mathrm{T}}\left(\mathcal{L} \otimes P^{-1}\right) \dot{\bar{x}}+\sum_{i=1}^{N} \frac{2}{\beta_{i}}\left(d_{i}-\bar{d}\right) \dot{d}_{i} \\
= & 2 \bar{x}^{\mathrm{T}}\left(\mathcal{L} \otimes P^{-1}\right)\left(\mathbf{I} \otimes A-\mathcal{L}_{e} \mathcal{D} \mathcal{L} \otimes P C_{y}^{\mathrm{T}} C_{y}\right) \bar{x} \\
& +2 \sum_{i=1}^{N}\left(d_{i}-\bar{d}\right)\left(\tilde{y}_{i}+C_{y} B_{r} \tilde{r}_{i}\right)^{\mathrm{T}}\left(\tilde{y}_{i}+C_{y} B_{r} \tilde{r}_{i}\right) \\
= & \bar{x}^{\mathrm{T}}\left[\mathcal{L} \otimes\left(P^{-1} A+A^{\mathrm{T}} P^{-1}\right)-2 \mathcal{L} \mathcal{D} \mathcal{L} \otimes C_{y}^{\mathrm{T}} C_{y}\right] \bar{x} \\
& +2 s^{\mathrm{T}}(\mathcal{L} \otimes \mathbf{I})\left[(\mathcal{D}-\bar{d} \mathbf{I}) \otimes\left[\begin{array}{ll}
\mathbf{I} & B_{r}
\end{array}\right] C_{y}^{\mathrm{T}} C_{y}\right. \\
& \left.\times\left[\begin{array}{ll}
\mathbf{I} & B_{r}
\end{array}\right]\right](\mathcal{L} \otimes \mathbf{I}) s .
\end{aligned}
$$

Similar to the equations in (14), there holds

$$
\begin{aligned}
s^{\mathrm{T}} & {\left[\mathcal{L}(\mathcal{D}-\bar{d} \mathbf{I}) \mathcal{L} \otimes\left[\begin{array}{ll}
\mathbf{I} & B_{r}
\end{array}\right]^{\mathrm{T}} C_{y}^{\mathrm{T}} C_{y}\left[\begin{array}{ll}
\mathbf{I} & B_{r}
\end{array}\right]\right] s } \\
= & s_{e}^{\mathrm{T}}\left[\mathcal{L}(\mathcal{D}-\bar{d} \mathbf{I}) \mathcal{L} \otimes\left[\begin{array}{ll}
\mathbf{I} & B_{r}
\end{array}\right]^{\mathrm{T}} C_{y}^{\mathrm{T}} C_{y}\left[\begin{array}{ll}
\mathbf{I} & B_{r}
\end{array}\right]\right] s_{e} \\
= & \bar{s}^{\mathrm{T}}\left[\mathcal{L}(\mathcal{D}-\bar{d} \mathbf{I}) \mathcal{L} \otimes\left[\begin{array}{cc}
\mathbf{I} & -B_{r} \\
0 & \mathbf{I}
\end{array}\right]^{\mathrm{T}}\left[\begin{array}{ll}
\mathbf{I} & B_{r}
\end{array}\right]^{\mathrm{T}} C_{y}^{\mathrm{T}} C_{y}\right. \\
& \left.\times\left[\begin{array}{ll}
\mathbf{I} & B_{r}
\end{array}\right]\left[\begin{array}{cc}
\mathbf{I} & -B_{r} \\
0 & \mathbf{I}
\end{array}\right]\right] \bar{s} \\
= & \bar{s}^{\mathrm{T}}\left[\mathcal{L}(\mathcal{D}-\bar{d} \mathbf{I}) \mathcal{L} \otimes\left[\begin{array}{cc}
\mathbf{I} & 0
\end{array}\right]^{\mathrm{T}} C_{y}^{\mathrm{T}} C_{y}\left[\begin{array}{ll}
\mathbf{I} & 0
\end{array}\right]\right] \bar{s} \\
= & \bar{x}^{\mathrm{T}}\left[\mathcal{L}(\mathcal{D}-\bar{d} \mathbf{I}) \mathcal{L} \otimes C_{y}^{\mathrm{T}} C_{y}\right] \bar{x} .
\end{aligned}
$$

From this equation, we have

$$
\dot{W}=\bar{x}^{\mathrm{T}}\left[\mathcal{L} \otimes\left(P^{-1} A+A^{\mathrm{T}} P^{-1}\right)-2 \bar{d} \mathcal{L}^{2} \otimes C_{y}^{\mathrm{T}} C_{y}\right] \bar{x} .
$$

Since the matrix $\mathcal{L}$ is symmetric, we can decompose it as $\mathcal{L}=$ $\left[N^{-1 / 2} \mathbf{1}, \mathcal{U}\right] \Lambda\left[N^{-1 / 2} \mathbf{1}, \mathcal{U}\right]^{\mathrm{T}}$, where $\left[N^{-1 / 2} \mathbf{1}, \mathcal{U}\right]$ is a unitary matrix and $\Lambda$ is a diagonal matrix with the eigenvalues of $\mathcal{L}$ on the diagonal. Particularly, the first entry of $\Lambda$ is zero. Let $\hat{x}=\operatorname{col}\left\{x_{1}, \ldots, x_{N}\right\} \triangleq\left[N^{-1 / 2} \mathbf{1}, \mathcal{U}\right]^{\mathrm{T}} \bar{x}$. Thus,

$\dot{W}=\bar{x}^{\mathrm{T}}\left[\left[N^{-1 / 2} \mathbf{1}, \mathcal{U}\right] \Lambda\left[N^{-1 / 2} \mathbf{1}, \mathcal{U}\right]^{\mathrm{T}} \otimes\left(P^{-1} A+A^{\mathrm{T}} P^{-1}\right)\right.$ 


$$
\begin{aligned}
& \left.-2 \bar{d}\left[N^{-1 / 2} \mathbf{1}, \mathcal{U}\right] \Lambda^{2}\left[N^{-1 / 2} \mathbf{1}, \mathcal{U}\right]^{\mathrm{T}} \otimes C_{y}^{\mathrm{T}} C_{y}\right] \bar{x} \\
= & \hat{x}^{\mathrm{T}}\left[\Lambda \otimes\left(P^{-1} A+A^{\mathrm{T}} P^{-1}\right)-2 \bar{d} \Lambda^{2} \otimes C_{y}^{\mathrm{T}} C_{y}\right] \hat{x} \\
= & \sum_{i=2}^{N} \lambda_{i}(\mathcal{L}) \hat{x}_{i}^{\mathrm{T}}\left(P^{-1} A+A^{\mathrm{T}} P^{-1}-2 \bar{d} \lambda_{i}(\mathcal{L}) C_{y}^{\mathrm{T}} C_{y}\right) \hat{x}_{i} .
\end{aligned}
$$

Let $\bar{d}$ be any constant such that $\bar{d} \geq \frac{1}{2} \lambda_{2}^{-1}(\mathcal{L})$. Then

$$
\begin{aligned}
\dot{W} & \leq \sum_{i=2}^{N} \lambda_{i}(\mathcal{L}) \hat{x}_{i}^{\mathrm{T}}\left(P^{-1} A+A^{\mathrm{T}} P^{-1}-C_{y}^{\mathrm{T}} C_{y}\right) \hat{x}_{i} \\
& =\sum_{i=2}^{N} \lambda_{i}(\mathcal{L}) \hat{x}_{i}^{\mathrm{T}} P^{-1} Q P^{-1} \hat{x}_{i} \leq 0 .
\end{aligned}
$$

Since $\dot{W}(t) \leq 0$ and $W(t) \geq 0, W(t)$ is bounded. Thus, $d_{i}(t)$ and $\mathcal{D}(t)$ are bounded. From the facts that $\dot{d}_{i}(t) \geq 0$ and $d_{i}(t)>0$, it follows that $d_{i}(t)$ converge to some finite positive constants. Moreover, because $\dot{W}(t) \leq 0$ and $\dot{W}(t)=0$ implies $\bar{x}(t)=0$, it follows from Lasalle's theorem that $\bar{x}(t) \rightarrow 0$ as $t \rightarrow \infty$. Consequently, consensus is reached under the protocol (5). The proof is completed.

The consensus conditions, Theorems 1 and 2, show that the consensus problem can be solved by the proposed adaptive output-feedback protocols (3) and (5), as long as the protocol gains are properly parameterized. Especially, designing the protocol gains only needs to know the system matrices of agents but does not need to compute the eigenvalues of the Laplacian matrix. The next natural question to ask is, whether the protocol gains that satisfy the specifications in the theorems are feasible under some common assumptions about the agents. This will be answered in the next subsection.

Remark 4: Theorems 1 and 2 can only deal with consensus on undirected graphs. Note that, even for state-feedback consensus control, adaptive protocols on directed graphs are quite different from those on undirected graphs (please refer to [15], [16] for related results). The main difficulty in extending Theorems 1 and 2 to directed graphs is due to the loss of symmetry of the Laplacian, which will make the previous derivations invalid. Designing reduced-order adaptive protocols on directed graphs deserves investigation in the future.

Remark 5: The consensus problem studied in this paper is leaderless. However, it is not difficult to extend Theorems 1 and 2 to the leader-follower case if the followers interact through some connected undirected graphs. To brief the edgebased case, let us denote the leader as $\dot{x}_{0}=A x_{0} ; y_{0}=C_{y} x_{0}$. Then the tracking error of agent $i$ is $e_{i}=y_{i}-y_{0}$. An edgebased reduced-order adaptive protocol can be constructed as

$$
\begin{aligned}
\dot{r}_{i}= & H r_{i}+F_{r} \sum_{j=1}^{N} c_{i j} a_{i j}\left(\tilde{y}_{i j}+C_{y} B_{r} \tilde{r}_{i j}\right) \\
& +c_{i 0} b_{i} F_{r}\left(e_{i}+C_{y} B_{r} r_{i}\right), \\
u_{i}= & G r_{i}+F_{u} \sum_{j=1}^{N} c_{i j} a_{i j}\left(\tilde{y}_{i j}+C_{y} B_{r} \tilde{r}_{i j}\right) \\
& +c_{i 0} b_{i} F_{u}\left(e_{i}+C_{y} B_{r} r_{i}\right),
\end{aligned}
$$

where $c_{i j}, i, j=1, \ldots, N$, are given by (4), $c_{i 0}$ are given by

$$
\dot{c}_{i 0}=\alpha_{i 0}\left(e_{i}+C_{y} B_{r} r_{i}\right)^{\mathrm{T}}\left(e_{i}+C_{y} B_{r} r_{i}\right)
$$

$$
c_{i 0}(0)>0, \alpha_{i 0}>0, i=1, \ldots, N,
$$

and $b_{i}$ is a positive constant if agent $i$ is connected to the leader, and $b_{i}=0$ otherwise. It can be verified that the lumped state $\varepsilon=\operatorname{col}\left\{\varepsilon_{1}, \ldots, \varepsilon_{N}\right\}$ with $\varepsilon_{i}=\operatorname{col}\left\{e_{i}, r_{i}\right\}$ satisfies

$$
\dot{\varepsilon}=\left[\mathbf{I} \otimes \tilde{A}+\left(\mathcal{C} \circ \mathcal{L}+\mathcal{C}_{0} \mathcal{B}\right) \otimes \tilde{B}\right] \varepsilon,
$$

where $\mathcal{C}_{0}=\operatorname{diag}\left\{c_{10}, \ldots, c_{N 0}\right\}$ and $\mathcal{B}=\operatorname{diag}\left\{b_{1}, \ldots, b_{N}\right\}$. By following the proof for the convergence of $s_{e}$ in (8), it can be verified that Statements 1) and 2) in Theorem 1 ensure the convergence of $\varepsilon$, implying that consensus is reached. A nodebased counterpart can also be constructed. Related details are omitted here for brevity.

\section{Design of Protocol Gains}

In this subsection, we discuss the feasibility of the protocol gains that are specified in Theorems 1 and 2. For simplicity, suppose that $B_{u}$ has full column rank; if this is not satisfied, one can extract the linearly independent columns of $B_{u}$ as the new input matrix and then repeat the design procedures. Motivated by [25], the following algorithm is proposed for designing the protocol gains of (3) and (5).

1) Select a matrix $J \in \mathbb{R}^{n_{x} \times\left(n_{x}-n_{u}\right)}$ such that $\left[\begin{array}{cc}B_{u} & J\end{array}\right]$ is nonsingular. Partition the inverse of $\left[\begin{array}{cc}B_{u} & J\end{array}\right]$ as

$$
\left.\left[\begin{array}{cc}
B_{u} & J
\end{array}\right]^{-1}=\left[\begin{array}{l}
S_{u} \\
S_{r}
\end{array}\right]\right\} n_{u} \text { rows } n_{x}-n_{u} \text { rows } .
$$

2) Compute the matrices $B_{r}, H$ and $G$ from the known matrices $J, S_{u}$ and $S_{r}$ that are obtained as above and another matrix $K \in \mathbb{R}^{n_{u} \times\left(n_{x}-n_{u}\right)}$ such that

$$
\begin{aligned}
H & =S_{r} A\left(J-B_{u} K\right) \text { and } H \text { is Hurwitz, } \\
B_{r} & =J-B_{u} K, G=\left(S_{u}+K S_{r}\right) A B_{r} .
\end{aligned}
$$

3) Compute the protocol gains $F_{u}$ and $F_{r}$ as

$$
F_{u}=-\left(S_{u}+K S_{r}\right) P C_{y}^{\mathrm{T}}, F_{r}=-S_{r} P C_{y}^{\mathrm{T}},
$$

where $P$ is given as in Theorem 1 .

Following the proof of [25, Theorem 2], it can be verified that the matrices obtained as above satisfy the specifications stated in the proposed consensus analysis conditions.

Remark 6: Some comments about the proposed protocols and the above algorithm are provided as follows:

1) The matrix $H$ is controlled by the variable $K\left(S_{r}\right.$ and $J$ are determined in the first step). It is known from [25, Remark 3] that the matrix pair $\left(S_{r} A J, S_{r} A B_{u}\right)$ are stabilizable, provided that $S_{r}$ and $J$ are given as above and the pair $\left(A, B_{u}\right)$ is stabilizable. Thus, by some standard design methods in linear control theory, it is easy to find a matrix $K$ such that $H$ is Hurwitz. Moreover, it is well known that the Riccati equation (11) is always feasible for some positive definite matrix $P$, since the pair $\left(A, C_{y}\right)$ is detectable. Consequently, it is seen that all the steps in the algorithm are feasible under the usual assumption about agents that $\left(A, B_{u}, C_{y}\right)$ are stabilizable and detectable.

2) Without accounting the laws of adaption, each local controller of the protocols designed by the algorithm is of 
$\left(n_{x}-n_{u}\right)$ th order (in view of $H \in \mathbb{R}^{\left(n_{x}-n_{u}\right) \times\left(n_{x}-n_{u}\right)}$ ). In this sense, the dynamic output-feedback protocols (3) and (5) resulting from this algorithm are some reduced-order protocols, which are computationally less demanding than the full-order adaptive dynamic outputfeedback ones in [15].

3) Theorems 1 and 2 are also applicable for designing fullorder protocols. For this case, it is more straightforward to find the protocol gains such that (9) and (10) are satisfied. Specifically, by directly selecting $B_{r}=-\mathbf{I}$ and $F_{u}=0$, the equation (9) reduces to $H=A+B_{u} G$ and the matrix $F_{r}$ in (10) is given by $F_{r}=P C_{y}^{\mathrm{T}}$. It is easy to see that the protocols (3) and (5) recover the fullorder ones in [15, (2) and (3)]. Thus, the results therein can be viewed as special cases of the proposed protocols and consensus analysis conditions in this paper.

4) By resorting to the results in [20], one could construct another kind of reduced-order adaptive protocols, for which a node-based one might be given by

$\dot{r}_{i}=H r_{i}+F y_{i}+T B_{u} u_{i}, u_{i}=d_{i} G K_{1} \tilde{y}_{i}+d_{i} G K_{2} \tilde{r}_{i}$

with $H \in \mathbb{R}^{\left(n_{x}-n_{y}\right) \times\left(n_{x}-n_{y}\right)}$. In no regard of the feasibility, an obvious drawback of the protocol, however, is the fact that it needs absolute output information about agents, so that it cannot be applied in some scenarios where only relative information between agents is available. On the contrary, the proposed protocols (3) and (5) do not need absolute output information. Another work about reduced-order protocols is [21], based on which one might overcome this issue. However the protocol therein needs relative input information between agents, which makes each controller require the information about neighbours' neighbours. On the contrary, the information flow in the protocols (3) and (5) is directly based on the communication graph.

5) It is well known that reduced-order output-feedback controllers are difficult to design in general. The reduced order of each local controller in the algorithm is set to $n_{x}-n_{u}$, so as to illustrate that both theorems always guarantees the existence of some reduced-order protocols under standard assumptions. However, it should be stressed that they do not exclude the possible existence of protocols that satisfy $n_{r}<n_{x}-n_{u}$. For instance, let

$A=\left[\begin{array}{lll}1 & 0 & 0 \\ 1 & 1 & 0 \\ 0 & 1 & 1\end{array}\right], B_{u}=\left[\begin{array}{c}7.4 \\ 11.1 \\ \frac{16.9}{3}\end{array}\right], C_{y}=\left[\begin{array}{l}0 \\ 0 \\ 1\end{array}\right]^{\mathrm{T}}$

A feasible solution satisfying (9) and (10) is as follows:

$$
\begin{aligned}
B_{r} & =\left[\begin{array}{l}
3.7 \\
3.7 \\
\frac{2.9}{3}
\end{array}\right], G=1, H=-1, F_{u}=F_{r}=-1, \\
P & =\left[\begin{array}{ccc}
61.5 & 51.3 & 11.1 \\
51.3 & 56.9 & 14.8 \\
11.1 & 14.8 & 6.6
\end{array}\right], \\
Q & =\left[\begin{array}{ccc}
0.21 & 0.18 & -0.24 \\
0.18 & 2.64 & 0.08 \\
-0.24 & 0.08 & 0.76
\end{array}\right] .
\end{aligned}
$$

Unfortunately, under the usual assumption that the agent matrices $\left(A, B_{u}, C_{y}\right)$ are stabilizable and detectable, there is no general, tractable procedure, like those in the algorithm, for checking the existence of a reduced-order protocol with $n_{r}<n_{r}-n_{u}$ and further re-constructing it. Except the case, $n_{r}=n_{x}-n_{u}$, as in the algorithm, checking and exploring such a reduced-order protocol should be on a case-by-case basis.

\section{Simulation EXAMPLE}

In this section, to illustrate the efficacy of the propose protocols, we provide an applied example on formation flying of spacecrafts. As in the [11], the problem setting is stated as follows. A group of spacecrafts are supposed to move in a circular orbit with a virtual spacecraft as the origin, while the linearized equations of relative dynamics of the $i$ th spacecraft are given by

$$
\dot{x}_{i}=\left[\begin{array}{cc}
0 & \mathbf{I}_{3} \\
A_{1} & A_{2}
\end{array}\right] x_{i}+\left[\begin{array}{c}
0 \\
\mathbf{I}_{3}
\end{array}\right] u_{i}
$$

where $x_{i}=\operatorname{col}\left\{p_{i}, \dot{p}_{i}\right\} \in \mathbb{R}^{6}, u_{i} \in \mathbb{R}^{3}$ and

$$
A_{1}=\left[\begin{array}{ccc}
0 & 0 & 0 \\
0 & 3 \omega_{0}^{2} & 0 \\
0 & 0 & -\omega_{0}
\end{array}\right], A_{2}=\left[\begin{array}{ccc}
0 & 2 \omega_{0} & 0 \\
2 \omega_{0} & 0 & 0 \\
0 & 0 & 0
\end{array}\right] \text {. }
$$

In the equation, $p_{i} \in \mathbb{R}^{3}$ and $\dot{p}_{i} \in \mathbb{R}^{3}$ are the relative position and velocity of the $i$ th-spacecraft in the $x, y, z$-axis of $3 \mathrm{D}$ space, and $\omega_{0}$ is the angular rate of the virtual spacecraft. Formation flying of spacecraft means $p_{i}-h_{i} \rightarrow p_{j}-h_{j}$ and $\dot{p}_{i} \rightarrow \dot{p}_{j}$, where $h_{i}-h_{j} \in \mathbb{R}^{3}$ denotes the desired, fixed relative position between spacecrafts $i$ and $j$. That is, the spacecrafts have the same final velocity while keeping a fixed formation.

Suppose that each spacecraft can measure the relative positive $p_{i}-p_{j}$ and know the desired position $h_{i}$ and $h_{j}$. Motivated by the edge-based protocol (3), the following distributed controllers are proposed for formation flying:

$$
\begin{aligned}
& \dot{r}_{i}=H r_{i}+F_{r} \sum_{j \in \mathcal{N}_{i}} c_{i j} a_{i j}\left(\tilde{y}_{i j}+C_{y} B_{r} \tilde{r}_{i j}\right), \\
& u_{i}=G r_{i}+F_{u} \sum_{j \in \mathcal{N}_{i}} c_{i j} a_{i j}\left(\tilde{y}_{i j}+C_{y} B_{r} \tilde{r}_{i j}\right)-A_{1} h_{i},
\end{aligned}
$$

where $c_{i j}$ is in (4), $\tilde{y}_{i j}=\left(p_{i}-h_{i}\right)-\left(p_{j}-h_{j}\right)$ and $H \in \mathbb{R}^{3 \times 3}$. Motivated by the node-based one (5), the following alternative distributed controllers can also be constructed:

$$
\begin{aligned}
& \dot{r}_{i}=H r_{i}+d_{i} F_{r} \sum_{j \in \mathcal{N}_{i}}\left(\tilde{y}_{i}+C_{y} B_{r} \tilde{r}_{i}\right), \\
& u_{i}=G r_{i}+d_{i} F_{u} \sum_{j \in \mathcal{N}_{i}}\left(\tilde{y}_{i}+C_{y} B_{r} \tilde{r}_{i}\right)-A_{1} h_{i},
\end{aligned}
$$

where $d_{i}$ is in (6), $\tilde{y}_{i}=\sum_{i=1}^{N} l_{i j}\left(p_{j}-h_{j}\right)$ and $H \in \mathbb{R}^{3 \times 3}$.

Remark 7: The idea of the reduced-order protocol in [20] is to use the absolute output to re-construct the absolute state of each agent. Thus, each local reduced-order controller therein is actually a reduced-order observer. However, rather than this physical meaning, the controller states in our protocols are just some intermediate variables for the control purpose, similar to most of the existing results like [11], [12], [15], [16]. 
Example 1: Consider a group of 4 spacecrafts which communicate with each other according to a line graph. Let the edge weights are all 1 . Thus, the Laplacian matrix is

$$
\mathcal{L}=\left[\begin{array}{cccc}
1 & -1 & 0 & 0 \\
-1 & 2 & -1 & 0 \\
0 & -1 & 2 & -1 \\
0 & 0 & -1 & 1
\end{array}\right]
$$

Let $\omega_{0}=0.005$. By the proposed algorithm, a feasible solution of the gains of the protocols (21) and (22) is given by

$$
\begin{aligned}
H & =-\mathbf{I}, B_{r}=\left[\begin{array}{c}
\mathbf{I} \\
-\mathbf{I}
\end{array}\right], G=\left[\begin{array}{ccc}
-1 & -0.01 & 0 \\
0.01 & -1 & 0 \\
0 & 0 & -1
\end{array}\right], \\
F_{u} & =\left[\begin{array}{ccc}
-2.7320 & -0.0058 & 0 \\
0.0058 & -2.7321 & 0 \\
0 & 0 & -2.7320
\end{array}\right], \\
F_{R} & =\left[\begin{array}{ccc}
-1.7320 & 0 & 0 \\
0 & -1.7321 & 0 \\
0 & 0 & -1.7320
\end{array}\right],
\end{aligned}
$$

and the matrix $P$, which is used to obtain the above $F_{u}$ and $F_{r}$ but is not presented here, is computed from the equation (11) with $Q=\mathbf{I}$. Note that, different from the results in [15], each local controller of the above protocols is only of 3rd order (in no regard of the laws of adaption). Thus, the proposed control protocols as above are less computationally demanding.

For simulation, we specify the desired positions as $h_{1}=$ $[-100 ; 0 ; 0], h_{2}=[-100 ; 0 ; 100], h_{3}=[0 ;-100 ; 100]$ and $h_{4}=[0 ;-100 ; 0]$. For the protocol $(21)$, let the scatars $\alpha_{i j}=$ 0.01 and the initial conditions $c_{i j}(0)=10^{-4}, i, j=1, \ldots$, For the protocol (22), let $\beta_{1}=0.01, \beta_{2}=0.02, \beta_{3}=0.03$ and $\beta_{4}=0.04$ and the initial conditions $d_{i}(0)=10^{-4}, i=$ $1, \ldots, 4$. The initial states of the protocols are set to zero. Figure 1 shows the simulation result under the above settings, where the initial conditions of the spacecrafts are not presented for saving space. It can be seen that the spacecrafts maintain the specified flying formation, while the adaptive gains $c_{i j}$ and $d_{i}$ converge to some positive constants. Thus, the effectiveness of the proposed theoretical results are clearly illustrated.

\section{CONCLUSION}

In this paper, the problem of fully distributed consensus control of linear MASs has been investigated, and novel reduced-order adaptive output-feedback protocols have been constructed and analyzed. The edge-based protocol associates each edge with a scalar gain that is adaptively updated by the output difference of the two agents on each edge, while the node-based one associates each agent with a scalar gain that is updated by the output difference sum of all neighbouring agents. Sufficient existence conditions have been derived and a design algorithm has been presented for the proposed protocols. It is shown that, under the common assumption that the agents are stabilizable and detectable, the proposed protocols with $\left(n_{x}-n_{u}\right)$ th-order local controllers must exist and can be easily found. Compared with the existing reducedorder protocols, the propose ones rely on relative output information of neighbouring agents only and can be designed and implemented in a fully distributed way. A simulation example on formation flying of spacecrafts has been provided for illustrating the efficacy of the proposed method.

\section{REFERENCES}

[1] S. Liu, L. Xie, and D. E. Quevedo, "Event-triggered quantized communication-based distributed convex optimization," IEEE Trans. Control Netw. Syst., vol. 5, no. 1, pp. 167-178, 2018.

[2] Y. Tang, H. Gao, and J. Kurths, "Distributed robust synchronization of dynamical networks with stochastic coupling," IEEE Trans. Circuits Syst. I, Reg. Papers, vol. 61, no. 5, pp. 1508-1519, 2014.

[3] Y. Tang, H. Gao, W. Zhang, and J. Kurths, "Leader-following consensus of a class of stochastic delayed multi-agent systems with partial mixed impulses," Automatica, vol. 53, pp. 346-354, 2015.

[4] J. Qin, C. Yu, and H. Gao, "Collective behavior for group of generic linear agents interacting under arbitrary network topology," IEEE Trans. Control Netw. Syst., vol. 2, no. 3, pp. 288-297, 2015.

[5] J. Qin, Q. Ma, Y. Shi, and L. Wang, "Recent advances in consensus of multi-agent systems: A brief survey," IEEE Trans. Ind. Electron., vol. 64, no. 6, pp. 4972-4983, 2017.

[6] S. Knorn, Z. Chen, and R. H. Middleton, "Overview: Collective control of multiagent systems," IEEE Trans. Control Netw. Syst., vol. 3, no. 4, pp. 334-347, 2016.

[7] H. A. Poonawala, A. C. Satici, H. Eckert, and M. W. Spong, "Collisionfree formation control with decentralized connectivity preservation for nonholonomic-wheeled mobile robots," IEEE Trans. Control Netw. Syst., vol. 2, no. 2, pp. 122-130, 2015.

[8] Z. Meng, D. V. Dimarogonas, and K. H. Johansson, "Attitude coordinated control of multiple underactuated axisymmetric spacecraft," IEEE Trans. Control Netw. Syst., vol. 4, no. 4, pp. 816-825, 2017.

[9] W. Xia, M. Cao, and K. H. Johansson, "Structural balance and opinion separation in trust-mistrust social networks," IEEE Trans. Control Netw. Syst., vol. 3, no. 1, pp. 46-56, 2016.

10] W. Ren and R. W. Beard, "Consensus seeking in multiagent systems under dynamically changing interaction topologies," IEEE Trans. Autom. Control, vol. 50, no. 5, pp. 655-661, May 2005.

[11] Z. Li, Z. Duan, G. Chen, and L. Huang, "Consensus of multiagent systems and synchronization of complex networks: A unified viewpoint," IEEE Trans. Circuits Syst. I, Reg. Papers, vol. 57, no. 1, pp. 213-224, Jan. 2010.

[12] X. Li, Y. C. Soh, and L. Xie, "Output-feedback protocols without controller interaction for consensus of homogeneous multi-agent systems: A unified robust control view," Automatica, vol. 81, pp. 37-45, 2017.

[13] X. Li, Y. C. Soh, L. Xie, and F. L. Lewis, "Cooperative output regulation of heterogeneous linear multi-agent networks via $H_{\infty}$ performance allocation," IEEE Trans. Autom. Control, vol. 64, no. 2, pp. 683-696, 2019.

[14] Z. Li, W. Ren, X. Liu, and M. Fu, "Consensus of multi-agent systems with general linear and Lipschitz nonlinear dynamics using distributed adaptive protocols," IEEE Trans. Autom. Control, vol. 58, no. 7, pp. 1786-1790, 2013.

[15] Z. Li, W. Ren, X. Liu, and L. Xie, "Distributed consensus of linear multiagent systems with adaptive dynamic protocols," Automatica, vol. 49, pp. 1986-1995, 2013.

[16] Z. Li, G. Wen, Z. Duan, and W. Ren, "Designing fully distributed consensus protocols for linear multi-agent systems with directed graphs," IEEE Trans. Autom. Control, vol. 60, no. 4, pp. 1152-1157, 2015.

[17] Y. Lv, Z. Li, Z. Duan, and J. Chen, "Distributed adaptive output feedback consensus protocols for linear systems on directed graphs with a leader of bounded input," Automatica, vol. 74, pp. 308-316, 2016.

[18] J. Sun, Z. Geng, Y. Lv, Z. Li, and Z. Ding, "Distributed adaptive consensus disturbance rejection for multi-agent systems on directed graphs," IEEE Trans. Control Netw. Syst., vol. 5, no. 1, pp. 629-639, 2018.

[19] H. Zhang, F. L. Lewis, and A. Das, "Optimal design for synchronization of cooperative systems: State feedback, observer and output feedback," IEEE Trans. Autom. Control, vol. 56, no. 8, pp. 1948-1952, Aug. 2011.

[20] Z. Li, X. Liu, P. Lin, and W. Ren, "Consensus of linear multi-agent systems with reduced-order observer-based protocols," Syst. Control Lett., vol. 60, pp. 510-516, 2011.

[21] B. Zhou, C. Xu, and G. Duan, "Distributed and truncated reduced-order observer based output feedback consensus of multi-agent systems," IEEE Trans. Autom. Control, vol. 59, no. 8, pp. 2264-2270, 2014. 


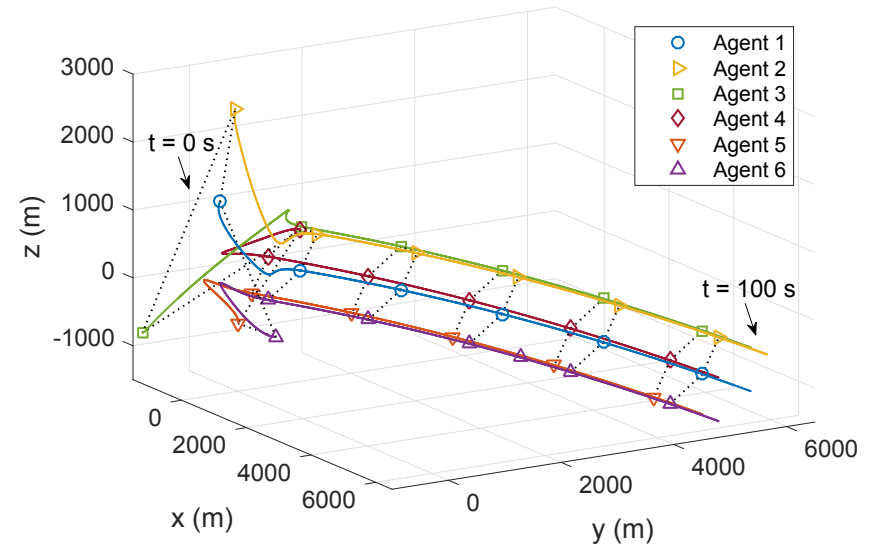

(a) Trajectories of spacecrafts under the edge-based protocol (21)

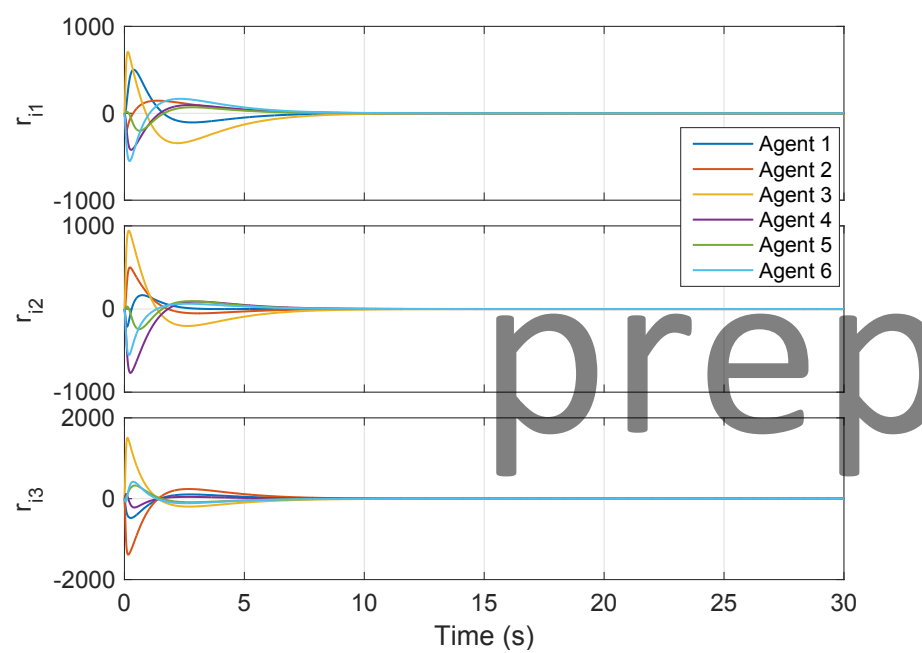

(c) State response of the edge-based protocol (21)

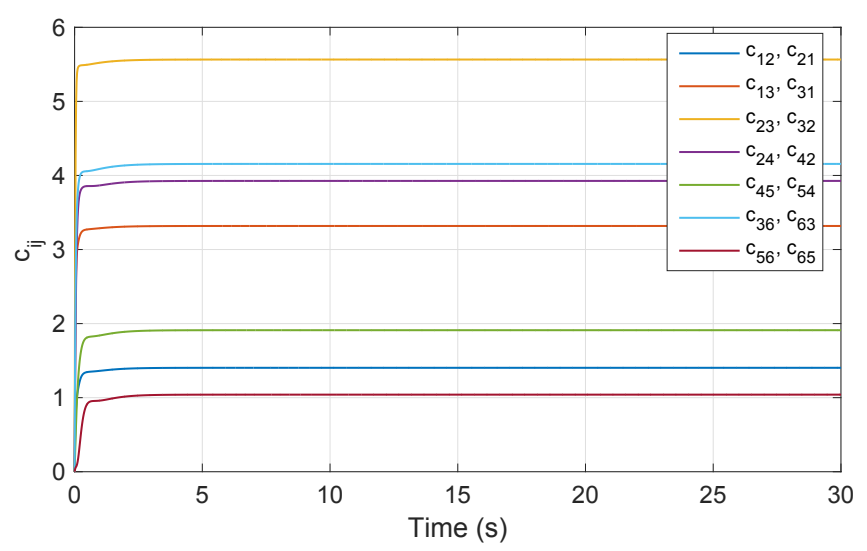

(e) $c_{i j}$ of the edge-based protocol (21)

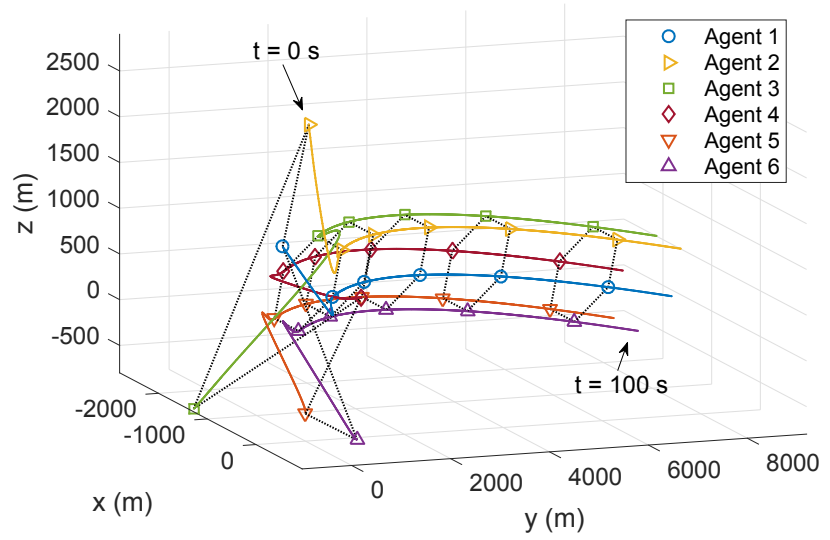

(b) Trajectories of spacecrafts under the node-based protocol (22)

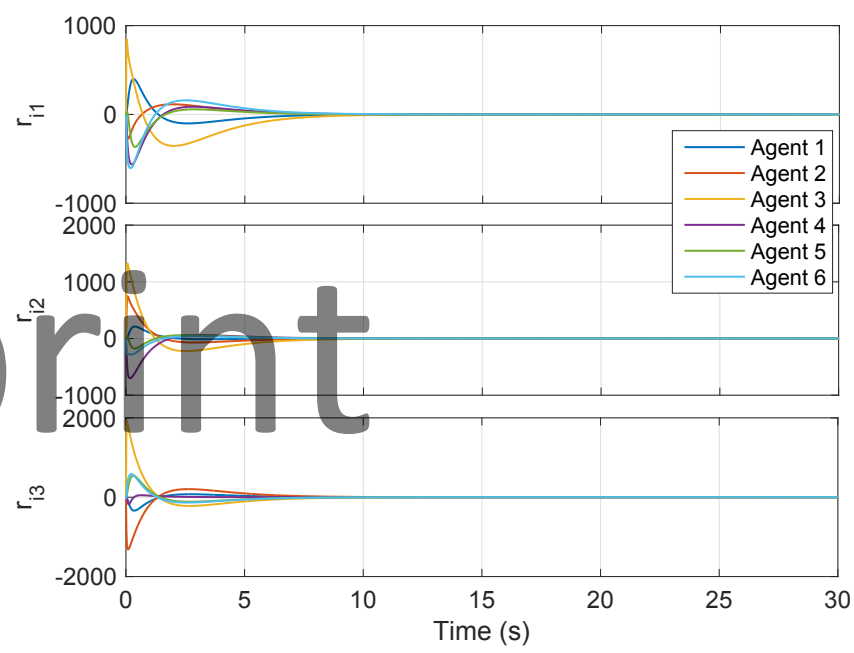

(d) State response of the node-based protocol (22)

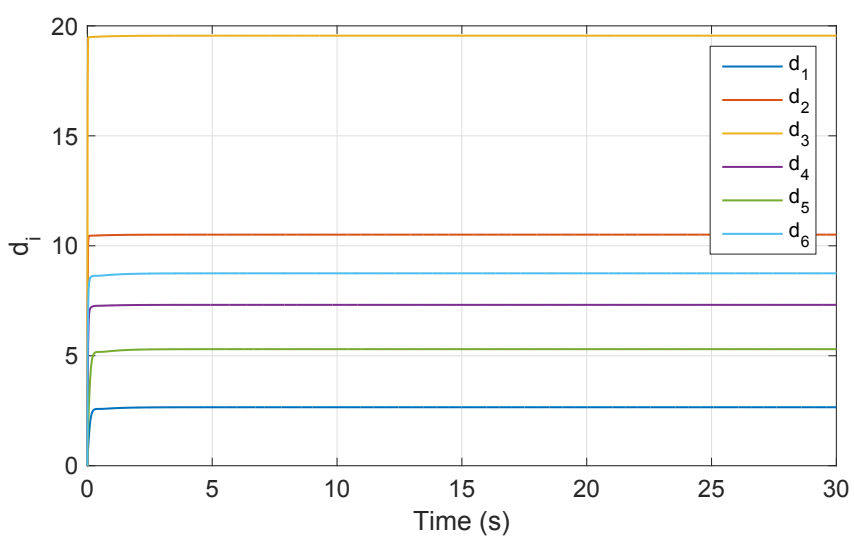

(f) $d_{i}$ of the node-based protocol (22)

Fig. 1. Simulation results 
[22] R. Olfati-Saber, "Flocking for multi-agent dynamic systems: Algorithms and theory," IEEE Trans. Autom. Control, vol. 51, no. 3, pp. 401-420, 2006.

[23] X. Li and S. Hirche, "Bounded consensus of linear multi-agent systems with external disturbances through a reduced-order adaptive feedback protocol," in 37th Chinese Control Conf., Wuhan, China, July 25-27 2018, pp. 6960-6965.

[24] H. K. Khalil, Nonlinear Systems, 3rd ed. Upper Saddle River, New Jersey: Prentice-Hall, 2002.

[25] X. Li, Y. C. Soh, and L. Xie, "A novel reduced-order protocol for consensus control of linear multi-agent systems," IEEE Trans. Autom. Control, vol. 64, no. 7, pp. 3005-3012, 2019.

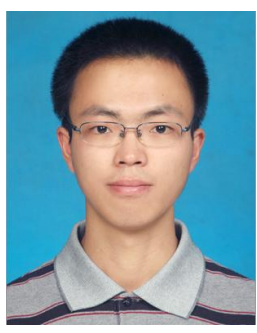

Xianwei Li (S'13-M'16) received the B.E. degree in Automation, the M.E. and Ph.D. degrees in Control Science and Engineering from Harbin Institute of Technology, Harbin, China, in 2009, 2011 and 2015, respectively. From September 2015 to September 2017, he was a Research Fellow with the School of Electrical and Electronic Engineering, Nanyang Technological University, Singapore. From October 2017 to March 2019, he was as an Alexander von Humboldt Research Fellow with the Department of Electrical and Computer Engineering, Technical University of Munich, Munich, Germany. Since April 2019, he has joined, as an assistant professor, the Department of Automation, Shanghai Jiao Tong University, Shanghai, China. His research interests include multi-agent systems, networked control systems, distributed control and robust control.

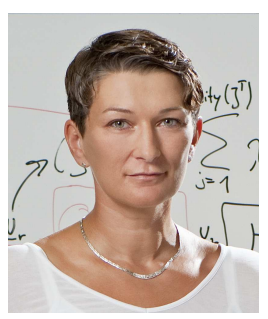

Sandra Hirche (M'03-SM'11) received the DiplomIngenieur degree in aeronautical engineering from Technical University Berlin, Germany, in 2002 and the Doktor-Ingenieur degree in electrical engineering from Technical University Munich, Germany, in 2005. From 2005 to 2007 she was awarded a Postdoc scholarship from the Japanese Society for the Promotion of Science at the Fujita Laboratory, Tokyo Institute of Technology, Tokyo, Japan. From 2008 to 2012 she has been an associate professor at Technical University Munich. Since 2013 she is TUM Liesel Beckmann Distinguished Professor and has the Chair of Information-oriented Control in the Department of Electrical and Computer Engineering at Technical University Munich.

Dr. Hirche's main research interests include cooperative, distributed and networked control with applications in human-machine interaction, multirobot systems, and general robotics. She has published more than 150 papers in international journals, books and refereed conferences. Dr. Hirche has served on the Editorial Boards of IEEE TRANSACTIONS ON CONTROL OF NETWORK Systems, IEEE TRANSACTIONS ON CONTROL Systems TEChNOLOGY, and IEEE TRANSACTIONS ON HAPTICS. She has received multiple awards such as the Rohde \& Schwarz Award for her PhD thesis, the IFAC World Congress Best Poster Award in 2005 and - together with students - the 2018 Outstanding Student Paper Award of the IEEE Conference on Decision and Control as well as Best Paper Awards of IEEE World Haptics and IFAC Conference of Manoeuvring and Control of Marine Craft in 2009.

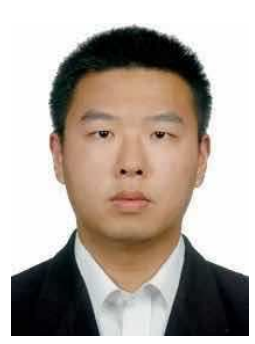

Fangzhou Liu (S'14) received the M.Sc. degree in control theory and engineering from Harbin Institute of Technology, Harbin, China, in 2014 and the Doktor-Ingenieur degree in electrical engineering from Technical University of Munich, Germany, in 2019. He is now a Research Fellow with the Chair of Automatic Control Engineering, Technical University Munich, Germany. His current research interests include networked control systems; modeling, analysis, and control on social networks; and their applications.

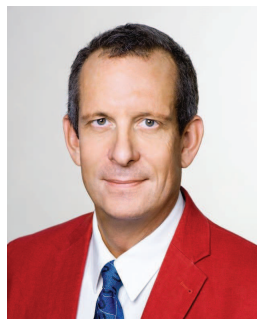

Martin Buss (F'14) received the Diploma Engineering degree from Technische Universität Darmstadt, Darmstadt, Germany, in 1990 and the Doctor of Engineering degree from The University of Tokyo, Tokyo, Japan, in 1994, both in electrical engineering.

In 1988, he was a Research Student for one year with Science University of Tokyo. From 1994 to 1995 , he was a Postdoctoral Researcher in the Department of Systems Engineering, Australian National University, Canberra, ACT, Australia. From 1995 to 2000, he was a Senior Research Assistant and Lecturer in the Chair of Automatic Control Engineering, Department of Electrical Engineering and Information Technology, Technical University of Munich,Munich, Germany. From 2000 to 2003, he was a Full Professor, the Head of the Control Systems Group, and the Deputy Director of the Institute of Energy and Automation Technology, Faculty IV, Electrical Engineering and Computer Science, Technical University Berlin, Berlin, Germany. Since 2003, he has been a Full Professor (Chair) in the Chair of Automatic Control Engineering, Faculty of Electrical Engineering and Information Technology, Technical University of Munich, where he has been in the Medical Faculty since 2008. Since 2006, he has also been the Coordinator of the Deutsche Forschungsgemeinschaft cluster of excellence "Cognition for Technical Systems (CoTeSys)," Bonn, Germany. His research interests include automatic control, mechatronics, multimodal human system interfaces, optimization, nonlinear, and hybrid discrete-continuous systems. 